\title{
Internet of things: why we are not there yet
}

\section{Salvatore Sorce* and Antonio Gentile}

\author{
Dipartimento di Ingegneria Chimica, \\ Gestionale, Informatica, Meccanica, \\ Università degli Studi di Palermo, \\ Viale delle Scienze, Edificio 6, \\ 90128 Palermo, Italy \\ and \\ InformAmuse S.r.1., \\ c/o Consorzio ARCA, \\ Viale delle Scienze, Edificio 16, \\ 90128 Palermo, Italy \\ E-mail: salvatore.sorce@unipa.it \\ E-mail: antonio.gentile@unipa.it \\ ${ }^{*}$ Corresponding author
}

\begin{abstract}
Twenty-one years past since Weiser's vision of ubiquitous computing (UbiComp) has been written, and it is yet to be fully fulfilled despite of almost all the needed technologies already available. Still, the widespread interest in UbiComp and the results in some of its fields pose a question: why we are not there yet? It seems we miss the 'octopus' head. In this paper, we will try to depict the reasons why we are not there yet, from three different points of view: interaction media, device integration and applications.
\end{abstract}

Keywords: ubiquitous computing; pervasive systems; interoperability; interaction media; device integration; human-environment interaction; internet of things.

Reference to this paper should be made as follows: Sorce, S. and Gentile, A. (2014) 'Internet of things: why we are not there yet', Int. J. Ad Hoc and Ubiquitous Computing, Vol. 16, No. 4, pp.232-239.

Biographical notes: Salvatore Sorce is an Assistant Professor at the Department of Chemical, Management, Computer, and Mechanical Engineering (DICGIM) of the University of Palermo. $\mathrm{He}$ holds the $\mathrm{PhD}$ in Computer Science and Engineering from the University of Palermo. His main research areas are pervasive and ubiquitous computing, novel human-computer interfaces, web and mobile technologies. He is a member of the ACM.

Antonio Gentile is an Associate Professor at the Department of Chemical, Management, Computer, and Mechanical Engineering (DICGIM) of the University of Palermo. He holds the $\mathrm{PhD}$ in Computer Engineering from the Georgia Institute of Technology (USA) and a $\mathrm{PhD}$ in Computer Science from the University of Palermo. His main research areas are human computer interaction and computer architecture. He is a senior member of the IEEE and of the ACM.

\section{Introduction}

It is now 21 years since Mark Weiser's seminal article about his vision of ubiquitous computing (UbiComp) has been written (Weiser, 1991). His leading idea was that "The most profound technologies are those that disappear. They weave themselves into the fabric of everyday life until they are indistinguishable from it"

According to Weiser's vision, a bunch of smart objects should be used to provide people with an artificial extension of the reality they live in and they interact with. People should thus interact with systems that should not be composed only by monolithic computers or bounded in a single place.

In fact, in the 'UbiComp' model, information processing is fully integrated within everyday objects and activities. Opposed to the desktop paradigm, in which people consciously use a single device for a specific purpose, those who use UbiComp engage many computational devices and systems simultaneously, and, better yet, they may not even be aware that they are doing so.

In the UbiComp paradigm, common objects are equipped with some additional features, such as I/O, computing, memory and networking capabilities, thus becoming 'smart'. This is the reason why this paradigm is 
often referred to as pervasive computing. The environment in which such objects are placed and used becomes 'augmented', providing people interacting within with features and capabilities that are not otherwise available.

A pervasive system is then made of a mix of heterogeneous devices such as sensors, actuators, handheld computers, mobile phones, RFID tags and readers, etc., somehow networked (often wirelessly). They must seamlessly react to changes occurring within the surrounding environment to provide services to their users. A pervasive system can, therefore, be seen as the physical implementation of UbiComp paradigm, and the underlying network of smart objects is often referred to as the internet of things.

The exponential diffusion of small and mobile smart devices, third-generation wireless communication devices, as well as location technologies, has led to a growing interest from the scientific community, the technical/ industrial world and the final users themselves, towards the great number of UbiComp-related fields and branches, as well as all of its possible real-life applications. In fact, a successful deployment of a pervasive system requires the collaboration of cross-disciplinary experts, such as hardware designers, wireless engineers, human-computer interaction specialists, software system developers, sociologists, application domain experts, and ultimately, users who will actually use the systems in their everyday life.

Clear evidence of this growing interest is the large number of specialised conferences, journals, special issues, magazines and transactions appeared in the 15 years, some of them becoming de facto reference for researchers worldwide (among them, the 'UbiComp' conference series, the IEEE 'Pervasive Computing' magazine, the Springer's 'Personal and Ubiquitous Computing' journal, the Elsevier's 'Pervasive and Mobile Computing' journal, the Inderscience 'International Journal of $\mathrm{Ad} \mathrm{Hoc} \mathrm{and}$ Ubiquitous Computing', to cite a few).

Scientists largely discussed about both software and hardware technologies to be studied, developed, implemented and advanced to be usefully involved within pervasive systems.

Notwithstanding this general interest, Weiser's vision is yet to be fully fulfilled, despite of almost all the needed technologies already available.

\section{Human-environment interaction}

As we mentioned earlier, pervasive systems are composed of a large variety of networked smart devices that supposedly enrich the environment they are deployed in. Devices devoted to processing logic should be mostly hidden to avoid their perception and prevent environment flooding (pervasive systems have not to be invasive). On the other hand, devices of a pervasive system devoted to implement the user interface cannot be hidden, or at least users have to be aware of them.

According to Mark Weiser's vision, the access to services provided by a pervasive system should be as more natural and 'unconscious' as possible. One of the best ways to describe how straightforward the interaction with a pervasive system should be is the eyeglasses metaphor. People affected by eyesight problems typically wear a pair of glasses (or - more recently - use contact lens that could be an even better metaphor). After a very short adaptation time, those people forget to wear glasses or lens, and they access the surrounding world as they do not have eyesight problems at all. At the same time, they act and move with no constraints, as they do not wear anything.

In the interaction design field, the focus is currently shifting from graphical user interfaces (GUIs) to natural user interfaces (NUIs), aiming at the translation of natural body movements into actions on the machine, thus eliminating the learning curve of a man-machine interface. This trend represents the first step towards the eyeglass metaphor, even if the underlying technology is not so 'disappearing'.

There are several attempts to design innovative devices, ways and modalities to implement more and more 'natural' human-environment interfaces, but the resulting interactions are not so natural (Malizia and Bellucci, 2012). People are often constrained to (consciously) use some device and to behave according to the interaction rules, which in turn are set by designers typically following constraints from the underlying technologies. Such rules frequently compel users to make unnatural gestures, actually restricting their freedom of action, in contrast with the eyeglasses metaphor.

In most cases, the proposed interaction modality seems to be more oriented towards showing of technological wonders rather than to the actual usability of the interface. In other words, interfaces are designed more to amaze people than to make them interact with the system in easy, intuitive ways.

\subsection{Lessons learnt in usability}

One of the lessons we learnt during our past activity in research and development of innovative and natural human-environment interaction is that the main focus must always be on the actual goal the deployed system is aimed at, forgetting all the astonishing media (if useless, of course). During a previous work carried out by our research group within the University and the R\&D staff of a university spin-off company, we have been involved in the implementation of an information provisioning system for a large wine fair in Italy (Vinitaly in Verona, 2011 and 2012 editions) (Gentile et al., 2011a, 2012; Pirrone et al., 2012). Our proposed solution provided people with multimodal access to the available services: a traditional point-and-click interface shown on a touch screen placed on the top of a totem-style case; a personal interface made available through an app to be installed on people's own smartphones; a short-range, self-positioning framework based on $\mathrm{QR}$ codes to quickly access information related to people's current position.

We tracked both the system usage and the people behaviour while searching for information, and we observed 
that the traditional point-and-click interaction mode was largely the most used one, with a ratio of 100:1 over the personal interface during the 2011 edition, raised to 1000:1 in 2012 (see Gentile et al. (2011a) and Pirrone et al. (2012) for more detailed reports). This was mainly due to the goal of the system, set according to the visitors' needs: to quickly find the preferred wine or producer, and locate it on the map with respect to their own position.

As a consequence of this analysis, we are reconsidering the opportunity to include a gesture-based interface we were designing in possible future deployments of the system in similar contexts. The expected results in terms of useful improvement could not be worth the needed efforts in terms of research and development, both in the human-environment interaction and hardware/software fields.

\subsection{Smartphones as unique interaction devices?}

To provide people with an adaptable device they are also used to interact with, the access to pervasive services is often mediated by personal mobile devices.

In recent years, there has been an increasing use of personal mobile devices (smartphones, PDAs and tablets) equipped with different wireless technologies, both for short and for long distance communications (RFID, Bluetooth, WiFi, ZigBee, UMTS, HSDPA, etc.), and showing intuitive interaction interfaces (touch screens and GUIs).

Mobile devices are widely available among people of all ages, and their diffusion is constantly increasing; they are almost in everyone's pocket and can be used almost anywhere (more than one billion smartphone users are estimated by 2014 - Gartner's studies). This distribution was also driven by the introduction of various types of sensors and multi-programmed operating systems. This justifies the common interest in the study of new ways of service provision (and brand-new services too) according to the features and capabilities of mobile devices (Berhe et al., 2004; Genco et al., 2006; La Cascia et al., 2010).

There are a large variety of application fields where services can be pervasively accessed by mobile devices, such as context-aware information provision within university campuses (Genco et al., 2005), interactive user profile-based guides in cultural heritage sites (Pilato et al., 2006; Raptis et al., 2005; Andolina et al., 2012), augmented reality objects assembly in mobility (Henrysson et al., 2005) and healthcare systems (Price and Summers, 2006; Vuong et al., 2009).

Users are allowed to install all the applications they consider interesting or useful, thus making personal devices an indispensable companion for both business or leisure tasks. This is one of the main reasons why users are more and more interested in such devices.

This opportunity has paved new ways to new business models for all those entities ranging from single individual programmers to large well-organised software houses, who are working on developing applications for these devices.

The great connectivity, the familiarity of interaction and the possibility to be programmed to perform many different tasks give personal mobile devices a chance to widely become 'intelligent terminals' to access any information system appropriately designed in a personalised and context-dependent way.

The large diffusion of smart personal devices also led to novel human-to-human interaction (HHI) modes (Gentile et al., 2011b). In fact, traditional interaction among humans takes place involving one or more of their five senses (touch, hearing, sight, smell and taste), as well as one or more of possible expressions (gesture and speech). Using one or more senses and expressions at a time obviously implies that people must be within the range of the involved sense(s) and expressions to interact one to another. For example, people must be in sight 1 to each other to interact by gesture.

The pressing communication needs and the increasing distances among people made HHIs to be more and more mediated by (a network of) some devices, thus leading to several social implications. For example, in contexts of face-to-face interaction, the surrounding environment - such as the temperature, the available furniture, the arrangement of the chairs and tables, and other external aspects - can affect the interaction. In a computer-mediated interaction, these have little or no importance, and the most relevant factors are linked to the perception of the counterpart given by the used media.

Smartphones can be considered as the terminals of the communication chain between two people, thus enabling them to cooperate for almost any task any time and any where.

The possibility given by our smartphones to access a large amount of information, such as that of the World Wide Web, or to read and write e-mail or instant messages, is UbiComp itself by definition. And, people use (and often abuse) this possibility more and more frequently, even when there is not a real reason to do it (Jarrow, 2012).

For all these reasons, one of the most popular uses of such devices is the access to personalised and on-demand information wherever it is needed. In fact, it has to be considered that human-environment interaction should not be the same for all, since differences in needs and skills of people have to be taken into account to avoid heavy compromises, which could not satisfy anyone. Because of their programmability and wide popularity, they can be made suitable to operate as remote controllers, or personal adaptive I/O interfaces, for applications remotely running. This way needed services can be accessed by means of a well-known device, with no need to learn how to use new kind of interface (Lukowicz et al., 2012).

Even if the allowed interfaces are not so natural and the resulting interaction modes with these devices are not as implicit as Weiser would have conceived, currently smartphones are perceived as the ubiquitous device above all others. Perhaps, they are perceived as invisible in the way they seem to blend into common actions and in how people have evolved practices around them (Schmidt et al., 2012). Anyway, their current large diffusion and its positive trend, along with their increasing capabilities, let us forecast significant leaps towards the common acceptance of 
such devices as interaction media in the next few years (Want, 2012).

\section{Device integration}

The 'augmented' environment resulting from the deployment of a pervasive system should be perceived in much the same way as we are used to manage our everyday life's reality, at any time and in any place, inside or outside our home or office.

There are many domains where pervasive systems are successfully exploited. One of the most recent and interesting applications of pervasive technology is the provision of advanced information services within public places, such as cultural heritage sites (Pilato et al., 2006; Raptis et al., 2005), or schools and university campuses (Genco et al., 2005). In such contexts, concurrent technologies exploited in smart mobile devices can be used to satisfy the mobility need of users allowing them to access relevant resources in a context-dependent manner. Of course, most of the constraints to be taken into account when designing a pervasive information providing system are given by the actual application domain where they are deployed. For example, it should be useless to provide people with a vocal interaction information system within a highly crowded place. In this case, the background noise could make the vocal interaction an actual handicap rather than being an aid.

Devices that can be used in a pervasive system may thus belong to different categories of price, size, portability, processing power, storage capacity, connectivity and I/O interfaces. Theoretically, pervasive systems could be made of any kind of device, whether they are equipped with sensors or actuators, or some processing capability, or even a subset of these features. As for the software, devices can be more or less programmable, or may be equipped with more or less interactive operating systems.

Because of the highly heterogeneous nature of the involved devices, the realisation of pervasive systems presents many challenges in cross-cutting areas of scientific research: integration, engineering, modelling, user interfaces, etc. Designers of pervasive systems have to take into account such heterogeneity (Niemelä and Latvakoski, 2004; Kang et al., 2012). Unfortunately, advances in such fields mostly moved independently one of each other. For this reason, although there are different implementations of pervasive systems for the provision of useful services, their design and integration through a common platform are still largely discussed issues within the scientific community. In fact, the integration of several devices of different kinds presents problems in terms of reliability, scalability, security, quality of service, privacy (Saha and Mukherjee, 2003) and service discovery (Thompson and Midkiff, 2013).

To provide the desired service, in most cases the devices must be programmed one by one (each with its specific programming language), and then be enabled to work together through some specific protocol. In fact, most of these devices have not been originally conceived to cooperate with others; they do not use a common language and often they do not have compatible interfaces. As a consequence, there is a need to create an infrastructure acting as a logical connection layer (which may be made of hardware, software, or both) among devices involved in pervasive systems.

Despite several efforts carried out by the scientific community towards the realisation of operating systems, programming languages, middleware and development platforms to support the interoperability among devices (Sorce et al., 2010), the achieved results are not yet satisfactory. This is mainly due to two factors:

- the different hardware and software technologies used were not designed to be closely integrated one to each other, and each of them has different control and interrogation interfaces

- $\quad$ there is a general lack in support by manufacturers that, except for some rare attempt not always been successful, tend to use ad-hoc solutions to retain their customers within brand-related communities.

This means that full interoperability is difficult to achieve, and current solutions narrow their focus on specific application domains by using specific devices. In other words, there is a big issue about the Thing-to-Thing interaction within the IoT. Processing and fusion of different distributed multimodal devices is still subject to widespread research (Lohr, 2013). The interfaces and services required are of unprecedented complexity and are being developed across a community of advanced research laboratories. As a consequence, there is the need to create an infrastructure acting as a logical connection layer (which may be made of hardware, software, or both) among devices involved in pervasive systems. This need is strongly felt by the international scientific community, as demonstrated by the recent proliferation of journals, magazines, special issues and conferences on this topic. Each of these venues attracts a large number of scientific publications concerning surveys (Jaroucheh et al., 2009) and proposed solutions (Bennaceur et al., 2009). However, most of the proposed solutions are tightly coupled with a specific class of systems/devices or with a given applicative domain, and no fully general solution has been proposed to the best of our knowledge.

In addition to academic research, recently a number of industry initiatives have been announced that try to address these very issues (Bennaceur et al., 2009). These initiatives are mostly vendor-centric, however, and focus on internetcentric solutions (i.e., the 'mobile as a client' approach).

One shared conclusion among scientists is that interoperability is required at all levels of UbiComp (Niemelä and Latvakoski, 2004). On the application level, mobile client applications must discover and interoperate with application services available to them at their present location. Such services are to be developed upon different middleware types and published using different service discovery protocols that are unknown to the application developer (Rybicki, 2008). This means that interoperability 
on the middleware level is also required. The mobility and availability of a huge number of heterogeneous resources at the same time entail further requirements such as scalability and resource-discovery (Issarny et al., 2007).

The role of middleware in such contexts is, therefore, essential to support mobility and adaptation of applications to the current context (Schmidt and Hauck, 2007; Ali and Crespi, 2012). A useful middleware should address some important issues. First, owing to the frequent disconnection and reconnection of mobile devices, it has to provide asynchronous communication. It must also be responsible for aggregating context information from different sources, storing them in appropriate format and providing querying and notification support. Such a middleware must present context to upper layers and communicate changes until the service layer is reached. In other words, a good middleware should be implemented in such a way as to achieve a tradeoff between transparency and awareness (Bellavista and Corradi, 2006).

Because of the large number of problems to be dealt with, designing and implementing pervasive systems is a complex task (Edwards et al., 2007). In particular, application adaptation based on context such as environmental factors, device limitations and connectivity requires the programmer to handle a complex combination of factors that manifest themselves throughout the application (Carton et al., 2007). As a consequence of the above-mentioned discussion, there are two conflicting trends:

- The growing interest towards the use of pervasive systems to provide people with useful services. This trend is leading to the study of new ways for service provision (and brand-new services too) according to the features and capabilities of such systems.

- The low device interoperability, which actually binds designers to set up a pervasive system taking into account all features of each involved device. Should the system layout need to be updated or even one of its components be replaced with a better one, the whole system needs to be redesigned and interactions redefined.

This is a big obstacle to the diffusion of pervasive systems, which otherwise would be the obvious solution for most situations where there is the need to provide people with information retrieved and presented in a context-dependent way.

\section{Applications}

There is a large scientific literature and, more recently, a lot of commercially available solutions dealing with the design and implementation of pervasive systems deployed in real-life environments to provide people with some kind of service.
According to Weiser's vision, the UbiComp paradigm should help people solve their everyday problems by means of an underlying framework of smart objects (the IoT) to be used in a natural, better if unconscious, way (remember the eyeglasses metaphor). The design and implementation process of a pervasive system could be outlined as follows:

- identification of a shared problem or need

- identification of required smart objects

- definition of the interaction modes and media.

If we exclude from this discussion, some example of really useful applications (even for niches of users, such as those devoted to the support of elderly, disabled, and, more generally, to healthcare), it seems that the actual process is reversed. The first step is to find an amazing interaction mode (the more trendy are gesture- or brain-based) to capture the attention of people, no matter what will be the actual goal of the system. The next step is the identification of the required smart objects, if available, or the set-up of new ones. The identification of a possible application of the composed system is the last step, thus leading to almost useless solutions, for two main reasons:

- the interaction media and modes often require a long learning time

- even if the interaction mode is natural, the interest for the application goal is limited to a very small number of people ("the availability of sensor-enabled shirts does not mean that a significant number of people will be regularly wearing them") (Schmidt et al., 2012).

The resulting systems are perceived as mere technologic exercises, with no practical usefulness.

\section{UbiComp: an octopus without the head}

As a support of the above-mentioned discussion, here we shortly report what happened during a post-doc workshop on pervasive systems and IoT within our department, where attendees presented their research activity to people with advanced skills in computer science, who did not work on the field. At the end of the presentation, one of the attendees argued that UbiComp seems to be an octopus without the head. More precisely, all UbiComp-related research and application fields seem to follow different development paths with no common goals. This generates a great confusion among common people, so that actually almost every information system based on a network that can be remotely accessed is (or could be) perceived to be a pervasive one.

One of the main reasons why there is not a clear understanding of what a pervasive system is so far, and also perhaps of what a pervasive system is not, is the lack of killer applications, such as the e-mail or the web for the internet. Most people will continue to wrongly identify the internet with the web (it is quite common to hear sentences like: "I read that on internet", when the actual meaning is: 
"I read that on a web page"), until it is not clear which is the container and which is the content.

According to recent experiments, it seems that pervasive advertising could become the first UbiComp's wide accepted application (Krumm, 2011), even if this seems to be opposite to what Weiser conceived as 'disappearing computer' (Schmidt et al., 2012). Initial studies show that, in fact, traditional advertising based on large printed posters can be successfully integrated with mobile and context-aware devices to create links between static and dynamic contents. Such integration would lead to personalised ads that will 'strike a balance between being calm when we do not need them and being engaged and inspired when we want to participate' (Müller et al., 2011).

Another promising field from which the killer app may come out is the service provision in public places, made accessible by smartphones. In fact, people always need information, directions and services when exploring an unknown place. The smartphone is the best way to allow users to quickly and easily access to the services provided by a system, even if never accessed before (see earlier). In particular, we are witnessing a rapid growth of cultural-heritage sites fruition by means of personal mobile devices. Instead of wear yet another device (audioplayers, videoguides or the like), visitors of such sites should simply connect their smartphone to the pervasive museum network and enjoy personalised interaction with exhibits and artefacts. The reason of the success of such applications is that interaction could take place in their own language, in really natural ways (Andolina et al., 2010), possibly using a level of detail and a vocabulary related to their own age or skills, thus making lasting memories of their visits.

In our opinion, HHI mediated by computers is, however, the best candidate to become the killer application, owing to its social relevance. In fact, there have been many studies that have analysed the dynamics of social groups that work or interact in different ways by means of some device (Driskell et al., 2003; Brown, 2000; Ruggieri, 2009, to cite a few). Their relevance is due to the strong empirical character of researches on computer mediated groups (CMGs), especially for those who design systems for virtual interaction, with the aim of optimising processes in function of the objectives to be achieved.

An example is represented by the learning and content management systems (LCMSs), which, in just a few years, have reached high levels of standardisation. This has created a new profession, the instructional designer, i.e., a system designer for training courses, who operates on online environments and contexts to achieve the best combination between the environment and the learning process.

\section{Conclusions}

A key problem with wider UbiComp adoption is the relationship with existing infrastructures, such as the cell phone network, power lines, and even smartphones and users themselves (Caceres and Friday, 2012). This has led to the research of new ways of possible exploitation of existing objects for other purposes with respect to those that were originally conceived for, such as smartphones. IoT is evolving and it is gaining a growing interest, being now the subject of several journals and magazines special issues, and of international conferences. There are a large number of scientific publications, end-user applications and people dealing with UbiComp-related fields and branches. Such a widespread interest in UbiComp and the results in some of its fields pose a question: why we are not there yet?

The above-mentioned discussion and the relevant literature suggest three possible answers:

- lack of really natural interfaces

- lack of interoperability

- lack of killer applications.

Despite this, it is still extraordinary how long forward Mark Weiser had foreseen the future when he wrote about his vision of UbiComp. We think that the key to the wide acceptance of pervasive systems based on the IoT rely on design factors, which are on the human side of the UbiComp vision, since the currently available technology could be enough to implement them.

When we will find the solution to all of the three issues listed earlier, people will 'wear' the IoT as eyeglasses to enhance their vision of the surrounding world.

\section{Acknowledgements}

The authors thank their colleagues at DICGIM and InformAmuse s.r.l., Salvatore Andolina, Antonio Massara, Dario Pirrone, Giuseppe Russo, Antonella Santangelo, and Eleonora Trumello, for their significant contribution by sharing ideas, brainstorming and offering (at times, contrasting) opinions.

This work has been supported by the Ministero dello Sviluppo Economico (Italian Ministry of Economic Development), Grant No. 00007M101, for the "Speaky Acutattile: una nuova piattaforma inclusiva di assistente intelligente vocale multicanale" research programme.

\section{References}

Ali, B.R. and Crespi, N. (2012) 'Profile context management in ubiquitous computing', Int. J. of Ad Hoc and Ubiquitous Computing, Vol. 11, No. 4, pp.237-245.

Andolina, S., Pirrone, D., Russo, G., Sorce, S. and Gentile, A. (2012) 'Exploitation of mobile access to context-based information in cultural heritage fruition', Seventh International Conference on Broadband, Wireless Computing, Communication and Applications (BWCCA 2012), 12-14 November, pp.322, 328.

Andolina, S., Santangelo, A. and Gentile, A. (2010) 'Adaptive voice interaction for $3 \mathrm{D}$ representation of cultural heritage site', 2010 International Conference on Complex, Intelligent and Software Intensive Systems (CISIS), 15-18 February, Krakow, pp.729-733. 
Bellavista, P. and Corradi, A. (2006) The Handbook of Mobile Middleware, Auerbach Publications, ISBN-10: 0849338336, ISBN-13: 978-0849338335.

Bennaceur, A., Singh, P., Raverdy, P-G. and Issarny, V. (2009) 'The iBICOOP middleware: enablers and services for emerging pervasive computing environments', IEEE International Conference on Pervasive Computing and Communications, PerCom 2009, 9-13 March, pp.1-6.

Berhe, G., Brunie, L. and Pierson, J.M. (2004) 'Modeling service-based multimedia content adaptation', Proceedings of the 1st ACM Conference on Computing Frontiers CF 2004, Ischia, Italy, pp.60-69.

Brown, R. (2000) Group Processes: Dynamics within and between Groups, 2nd ed., Blackwell, Oxford.

Caceres, R. and Friday, A. (2012) 'Ubicomp systems at 20: progress, opportunities, and challenges', IEEE Pervasive Computing, Vol. 11, No. 1, January-March, pp.14-21.

Carton, A., Clarke, S., Senart, A. and Cahill, V. (2007) 'Aspectoriented model-driven development for mobile context-aware computing', Proceedings of the 1st International Workshop on Software Engineering for Pervasive Computing Applications, Systems, and Environments (SEPCASE '07), IEEE Computer Society, Minneapolis, MN, p.5, doi: 10.1109/ SEPCASE.2007.3.

Driskell, J.E., Radtke, P.H. and Salas, E. (2003) 'Virtual teams: effects of technological mediation on team performance', Group Dynamics: Theory, Research, and Practice, Vol. 7, pp.287-323.

Edwards, G., Seo, C., Popescu, D., Malek, S. and Medvidovic, N. (2007) 'Self-* software architectures and component middleware in pervasive environments', Proceedings of the 5th International Workshop on Middleware for Pervasive and Ad-hoc Computing (MPAC 2007), held at the ACM/IFIP/USENIX 8th International Middleware Conference, 26-30 November, Newport Beach, California, USA, pp.25-30.

Genco, A., Sorce, S., Reina, G. and Santoro, G. (2006) 'An agentbased service network for personal mobile devices', IEEE Pervasive Computing, Vol. 5, No. 2, April-June, pp.54-61.

Genco, A., Sorce, S., Reina, G., Santoro, G., Messineo, R., Raccuglia, R., Lo Vecchio, L. and Di Stefano, G. (2005) 'An augmented campus design for context-aware service provision', Proceedings of the 33rd annual ACM SIGUCCS Conference on User Services, 6-11 November, Monterey, CA, pp.92-97.

Gentile, A., Andolina, S., Massara, A., Pirrone, D., Russo, G., Santangelo, A., Trumello, E. and Sorce, S. (2011b) 'A multichannel information system to build and deliver rich user-experiences in exhibits and museums', 2011 International Conference on Broadband and Wireless Computing, Communication and Applications (BWCCA), 26-28 October, Technical University of Catalonia, Barcelona, Spain, pp.57-64, DOI: 10.1109/BWCCA.2011.14.

Gentile, A., Andolina, S., Massara, A., Pirrone, D., Russo, G., Santangelo, An., Trumello, E. and Sorce, S. (2012) 'QRouteMe: a multichannel information system to ensure rich user-experiences in exhibits and museums', Journal of Telecommunications and Information Technology (JTIT), Vol. 1, pp.58-66.

Gentile, A., Santangelo, A., Sorce, S. and Vitabile, S. (2011a) 'Human-to-human interfaces: emerging trends and challenges', International Journal of Space-Based and Situated Computing (IJSSC), Vol. 1, No. 1, pp.3-17.
Henrysson, A., Ollila, M. and Billinghurst, M. (2005) 'Mobile phone based AR scene assembly', Proceedings of the 4th International Conference on Mobile and Ubiquitous Multimedia, Christchurch, New Zealand, pp.95-102.

Issarny, V., Caporuscio, M. and Georgantas, N. (2007) 'A perspective on the future of middleware-based software engineering', Future of Software Engineering, 2007. FOSE '07, 23-25 May, pp.244-258.

Jaroucheh, Z., Liu, X. and Smith, S. (2009) 'A perspective on middleware-oriented context-aware pervasive systems', 33rd Annual IEEE International Computer Software and Applications Conference, COMPSAC '09, 20-24 July, Vol. 2, pp.249-254.

Jarrow, C. (2012) 10 Ways to Be Smarter about Your Smartphone, http://timemanagementninja.com/2012/04/10 ways-to-be-smarter-about-your-smartphone/ (last visit 22 April, 2013).

Kang, E.Y., Park, H. and Chae, J. (2012) 'A hybrid message delivery scheme for improving service discovery in mobile ad-hoc networks', Journal of Internet Technology, Vol. 13, No. 6, pp.879-890.

Krumm, J. (2011) 'Ubiquitous advertising: the killer application for the 21st century', IEEE Pervasive Computing, Vol. 10, No. 1, pp.66-73.

La Cascia, M., Morana, M. and Sorce, S. (2010) 'Mobile interface for content-based image management', Third International Workshop on Intelligent Interfaces for Human-Computer Interaction (IIHCI-2010), 15-18 February, pp.718-723.

Lohr, S. (2013) 'A messenger for the internet of things', Bits, the Business of Technology, 24 April, NYTtimes.com, http://bits.blogs.nytimes.com/2013/04/25/a-messenger-for-the -internet-of-things/ (last visit 30 April, 2013).

Lukowicz, P., Pentland, S. and Ferscha, A. (2012) 'From context awareness to socially aware computing', Pervasive Computing, IEEE, Vol. 11, No. 1, January-March, pp.32-41.

Malizia, A. and Bellucci, A. (2012) 'The artificiality of natural user interfaces', Commun. ACM, Vol. 55, No. 3, March, pp.36-38.

Müller J., Alt, F. and Michelis, D. (2011) 'Pervasive advertising', Human-Computer Interaction Series 2011, Springer, London, pp.1-29, doi: 10.1007/978-0-85729-352-7.

Niemelä, E. and Latvakoski, J. (2004) 'Survey of requirements and solutions for ubiquitous software', Proceedings of the $3 \mathrm{rd}$ International Conference on Mobile and Ubiquitous Multimedia (MUM '04), ACM, NY, USA, pp.71-78.

Pilato, G., Augello, A., Santangelo, A., Gentile, A. and Gaglio, S. (2006) 'An Intelligent Multimodal Site-guide for the Parco Archeologico della Valle dei Templi in Agrigento', Proc. of First European Workshop on Intelligent Technologies for Cultural Heritage Exploitation at The 17th European Conference on Artificial Intelligence, Riva del Garda, Italy, August, pp.45-49.

Pirrone, D., Andolina, S., Santangelo, A., Gentile, A. and Takizava, M. (2012) 'Platforms for human-human interaction in large social events', Seventh International Conference on Broadband, Wireless Computing, Communication and Applications (BWCCA), 2012, 12-14 November, 2012, Victoria, BC, pp.545-551.

Price, S. and Summers, R. (2006) 'Mobile healthcare in the home environment', Proceedings of 28th Annual International Conference of the IEEE Engineering in Medicine and Biology Society EMBS '06, August, New York, NY, pp.6446-6448. 
Raptis, D., Tselios, N. and Avouris, N. (2005) 'Context-based design of mobile applications for museums: a survey of existing practices', Proceedings of the 7th ACM International Conference on Human-Computer Interaction with Mobile Devices \& Services, Salzburg, Austria, pp.153-160.

Ruggieri, S. (2009) 'Leadership in virtual teams: a comparison of transformational and transactional leaders', Social Behavior and Personality, Vol. 37, No. 7, pp.1017-1022.

Rybicki, T. (2008) 'Semantic service discovery in pervasive computing environment', Proceedings of the 5th International Conference on Pervasive Services (ICPS '08), ACM, NY, USA, pp.69-72.

Saha, D. and Mukherjee, A. (2003) 'Pervasive computing: a paradigm for the 21st century', IEEE Computer, March, Vol. 36, No. 3, pp.25-31.

Schmidt, A., Pfleging, B., Alt, F., Sahami, A. and Fitzpatrick, G. (2012) 'Interacting with 21st-century computers', Pervasive Computing, IEEE, Vol. 11, No. 1, January-March, pp.22-31.

Schmidt, H. and Hauck, F.J. (2007) 'SAMProc: middleware for self-adaptive mobile processes in heterogeneous ubiquitous environments', Proceedings of the 4th Middleware Doctoral Symposium (MDS '07), ACM, Article 11, 6 pages.
Sorce, S., Augello, A., Santangelo, A., Gentile, A., Genco, A., Gaglio, S. and Pilato, G. (2010) 'Interacting with augmented environments', Pervasive Computing, IEEE, Vol. 9, No. 2, April-June, pp.56-58.

Thompson, M.S. and Midkiff, S.F. (2013) 'Service description and dissemination for service discovery in pervasive computing environments', Int. J. of Ad Hoc and Ubiquitous Computing, Vol. 12, No. 4, pp.193-204.

Vuong, N.K., Chan, S. and Lau, C.T. (2009) 'Classification of $\mathrm{pH}$ levels using a mobile phone', Proceedings of the 13th IEEE International Symposium on Consumer Electronics (ISCE2009), Kyoto, Japan, pp.823-827.

Want, R. (2012) 'Just a few more years', in Nigel Davies' 'Twenty Years On', Pervasive Computing, IEEE, Vol. 11, No. 1, January-March, pp.2-4.

Weiser, M. (1991) 'The computer for the 21st century', Scientific Am., Vol. 265, No. 3, pp.66-75. 